\title{
A neutral hydrogen survey of polar ring galaxies
}

\section{Parkes observations ${ }^{\star}$}

\author{
W. van Driel ${ }^{1}$, F. Combes $^{2}$, M. Arnaboldi ${ }^{3}$, and L. S. Sparke ${ }^{4}$ \\ 1 GEPI, Observatoire de Paris, Section de Meudon, 5 place Jules Janssen, 92195 Meudon, France \\ 2 LERMA, Observatoire de Paris, 61 avenue de l'Observatoire, 75014 Paris, France \\ e-mail: francoise.combes@obspm.fr \\ 3 Osservatorio Astronomico di Capodimonte, V. Moiariello 16, Napoli 80131, Italy \\ e-mail: magda@cerere.na.astro.it \\ 4 Astronomy Department, University of Wisconsin-Madison, 475 N. Charter St., Madison WI 53706, USA \\ e-mail: sparke@uwast.astro.wisc.edu
}

Received 13 December 2001 / Accepted 5 February 2002

\begin{abstract}
A total of 33 polar ring galaxies and polar ring galaxy candidates were observed in the 21-cm $\mathrm{H} \mathrm{I}$ line with the 64-m Parkes radio telescope. The objects, selected by their optical morphology, are all south of declination $-39^{\circ}$ and in only 5 of them $\mathrm{H}$ i had been reported previously. H I line emission was detected towards 18 objects, though in 3 cases the detection may be confused by another galaxy in the telescope beam, and one is a marginal detection. Eight objects were detected for the first time in $\mathrm{HI}$, of which 5 did not have previously known redshifts.
\end{abstract}

Key words. galaxies: distances and redshifts - galaxies: general - galaxies: ISM - radio lines: galaxies

\section{Introduction}

A polar-ring galaxy (hereafter referred to as PRG) consists of a flattened galaxy with an outer ring of gas, dust, and stars rotating in a plane approximately perpendicular to the central disc. Kinematically confirmed PRGs with a disc-dominated central galaxy tend to have wide, extended polar rings, while bulge-dominated objects show only short, narrow rings (Whitmore 1991; Reshetnikov \& Sotnikova 1997). The PRGs probably represent merger products, and their study may give us valuable clues about the process and frequency of merging; their visible environment appears to be similar to that of normal galaxies (Brocca et al. 1997), which may support long formation and evolution times of the rings. In addition, measurement of rotation in the two nearly perpendicular planes of the ring and galaxy provide one of the few available probes of the three-dimensional shape of galactic gravitational potentials, and hence the shape of dark and luminous matter distributions.

The catalogue of Whitmore et al. (1990, hereafter PRC) provides us with over a hundred known polar-ring

Send offprint requests to: W. van Driel,

e-mail: wim.vandriel@obspm.fr

* Table 1 is also available in electronic form at the CDS via anonymous ftp to cdsarc.u-strasbg.fr (130.79.128.5) or via

http://cdsweb.u-strasbg.fr/cgi-bin/qcat?J/A+A/386/140 galaxies and PRG candidates, as well as a list of possibly related systems, divided into the four main categories listed below. The updated number of objects per category listed here is different from that tabulated originally in the PRC, as since its publication the following 4 category $\mathrm{B}$ and 4 category $\mathrm{C}$ objects have been promoted to category A: B-03 (=IC 1689), B-17 (=UGC 9562), B-19 (=AM 2020-504), B-21 (=ESO603G21), C-13 (=NGC 660), C-24 (=UGC 4261), C-27 (=UGC 4385) and C-45 (=NGC 5128); see references in Paper III. All of these will be considered members of the A category in our studies - note that of these objects only B-19 was observed in our present Parkes survey.

1. A: kinematically-determined Polar-Ring Galaxies (17 objects)

2. B: good candidates for Polar-Ring Galaxies (20 objects)

3. C: possible candidates for Polar-Ring Galaxies (69 objects)

4. D: systems possibly related to Polar-Ring Galaxies (51 objects).

The scientific goals of our 21-cm H I line surveys of PRGs are to:

1. Establish redshifts for the objects with previously unknown redshift. 
2. Measure the amount of neutral hydrogen in these systems and examine its correlations with other observational parameters.

3. Identify objects for subsequent synthesis mapping; H I maps together with optical line studies will show which of the new morphological candidates are true polarring galaxies, and high-resolution maps will allow dynamical modelling.

Detailed observations of PRGs in the 21-cm HI line with large radio synthesis telescopes like the Australia Telescope, VLA or Westerbork, are crucial for an understanding of the dynamical state of these systems. Such mapping measures the distribution and the velocity field of gas in the rings, both of which are required for accurate determination of the shape of the dark halo. Together with optical absorption-line studies of the central galaxy, rotation in the ring gas determines whether morphological candidates are true polar rings. Further, knowledge of the ring mass is necessary to assess the stability of the rings against differential precession, an important consideration in estimating the time since its formation.

After having observed a sample of 47 PRC objects in the 21-cm Hi line with the 140-foot (43-m) Green Bank telescope (Paper I), 44 PRC objects with the 100-m Effelsberg telescope (Huchtmeier 1996, Paper II) and 50 PRC objects with the 100-m class Nançay telescope (van Driel et al. 2000, Paper III), we now present observations of 33 PRC objects in the southern hemisphere with the 64-m Parkes telescope. Provisional results of a pilot PRG H I survey made at Parkes in 1992 by a member of our team, O.-G. Richter (private communication), were refered to in Paper III.

In Sect. 2 of the present paper the sample of PRGs observed in $\mathrm{H}$ I at Parkes is presented. The observations are described in Sect. 3, a brief discussion of the results is given in Sect. 4 and the conclusions are summarized in Sect. 5. An analysis of these, and all other available $\mathrm{HI}$ data on PRGs and related objects will be presented in Paper V in these series (van Driel et al. in preparation).

\section{The Parkes polar-ring galaxy sample}

Listed in Table 1 are basic data for all 33 galaxies observed for our PRG HI survey at Parkes. These data, compiled from many sources, by no means form a homogeneous set. The sources used, in order of preference, are the online Lyon-Meudon Extragalactic Database (LEDA) http://leda.univ-lyon1.fr, the NASA/IPAC Extragalactic Database (NED) http:// nedwww.ipac.caltech.edu and the PRC. Generally, the data listed are mean, corrected values from the LEDA database, while the data in parentheses are uncorrected published values compiled in NED. Note that all radial velocities listed in this paper are heliocentric and calculated according to the conventional optical definition $\left(V=\mathrm{c}\left(\lambda-\lambda_{0}\right) / \lambda_{0}\right)$. The total blue magnitudes, $B_{\mathrm{T}}$ are sometimes indicative only, as they cannot be assumed to be on the same photometric scale, nor do all represent the true total apparent blue magnitude as defined in, e.g., the RC3; sometimes a magnitude measured within a single aperture is used instead. Neither do the isophotal diameters, $D_{25}$, represent a homogeneous set of measurements, as they sometimes refer to the size of the faint polar ring, and sometimes to the size of the brighter equatorial disc.

Of the 33 PRC objects observed at Parkes, 28 were selected using the following criteria: (1) South of declination $-39^{\circ}$ (the limit of our Nançay survey; our Green Bank survey reached $-45^{\circ}$ ), (2) from PRC Categories A, B or C , and (3) no published $\mathrm{H}$ I detection and not detected in the Parkes HIPASS Public Data Release spectra http://www.atnf.csiro.au/ research/multibeam/release/. Of these 28, 14 have optical redshifts, 5 of which (of B-25, B-26, B-27, C-65 and $\mathrm{C}-72$ ) are outside the range of the HIPASS spectra, -1281 to $12741 \mathrm{~km} \mathrm{~s}^{-1}$.

Besides the 28 objects thus selected, the telescope time allocation also permitted us to observe the following 6 objects: three (PRC A-05 = NGC 4650A, C-11= NGC 625 and C-42 = NGC 4672) that were detected in HIPASS and previously reported as detected in the literature, two (C-15 = ESO 199-IG12 and C-55 = ESO 143-G37) that were detected in HIPAS, and one (C-14 = NGC 979) whose previously reported detection seems due to radio interference. For details on the available $\mathrm{H}$ I data for all objects we refer to Table 2 and Sect. 4.1.

\section{Observations}

The observations with the Parkes 64-m telescope were made in August 2001, using about 75 hours of telescope time. The data were obtained with the 13-element 21-cm Multibeam receiver (Staveley-Smith et al. 1996), of which the inner 7 elements only were consecutively pointed towards the target galaxy. The $F W H M$ of the telescope in the observing mode used at 21-cm wavelength is 14.4 . The autocorrelator was divided into two pairs of cross-polarized receiver banks, each with 2048 channels and a $64 \mathrm{MHz}$ bandpass, giving a radial velocity coverage of about $12500 \mathrm{kms}^{-1}$ and a channel separation of $6.6 \mathrm{~km} \mathrm{~s}^{-1}$. For objects with known redshifts, the centre frequencies of the two banks were generally tuned to an $\mathrm{H}$ I velocity of $5500 \mathrm{~km} \mathrm{~s}^{-1}$ or $15000 \mathrm{~km} \mathrm{~s}^{-1}$, depending on the redshift. For the 3 objects (PRC B-18, C-22 and $\mathrm{C}-48$ ) with redshifts in the overlapping region, 9000 to $12000 \mathrm{~km} \mathrm{~s}^{-1}$, a centre velocity of $10000 \mathrm{~km} \mathrm{~s}^{-1}$ was used. For objects of unknown redshift, line searches were first made in the -1000 to $12000 \mathrm{~km} \mathrm{~s}^{-1}$ range and, if not detected, in the $8500-21500 \mathrm{~km} \mathrm{~s}^{-1}$ range; the two rms. noise levels listed in Table 1 for undetected objects refer to these two velocity search ranges. The observing time was distributed as equally as possible between the different line searches, about 1.25 hours per velocity range per object, hence the similarity in rms noise levels. 


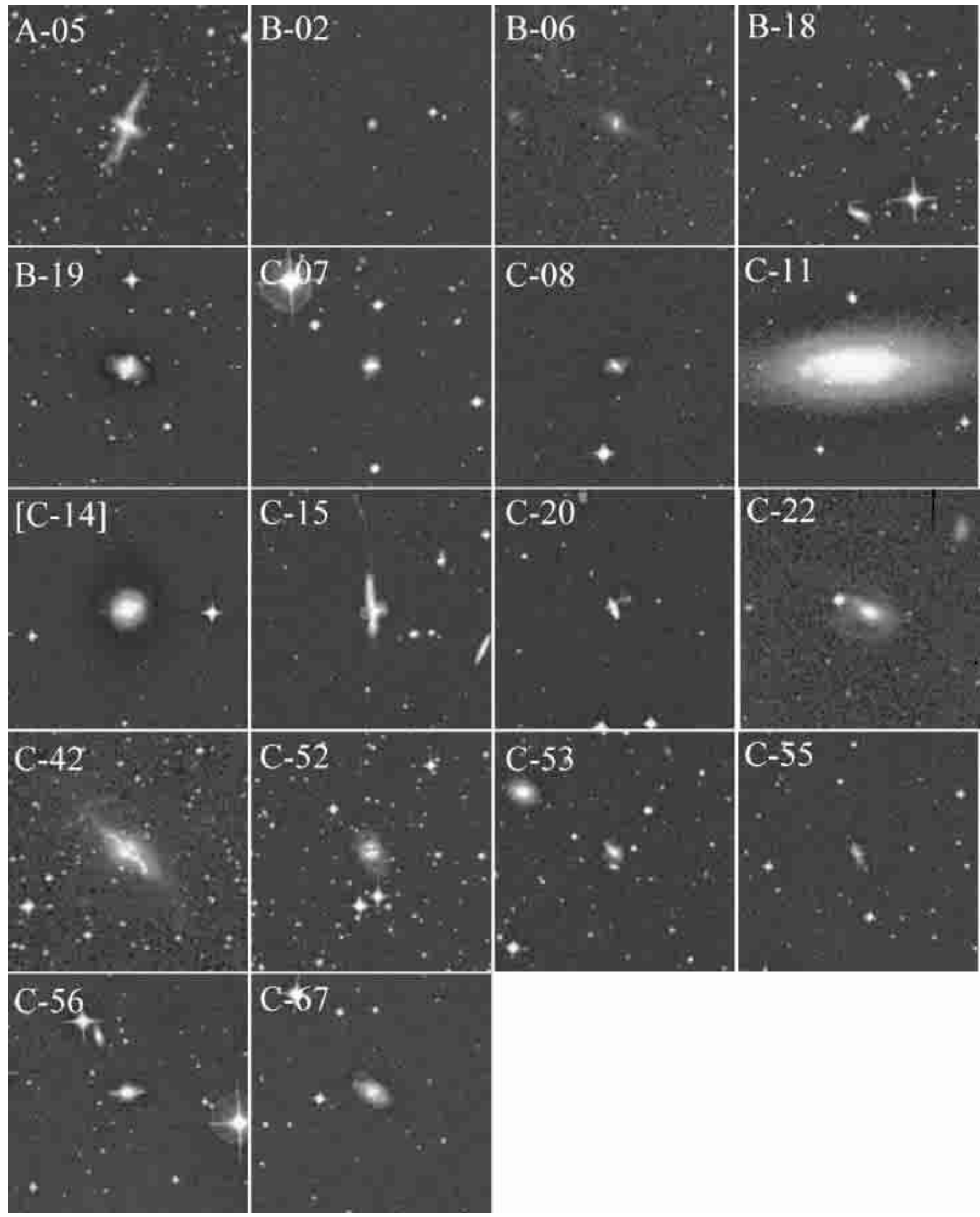

Fig. 1. Optical Digital Sky Survey images of all 18 PRC objects towards which H i line emission was detected at Parkes. Each galaxy is identified by its PRC name. Each image has a size of $5^{\prime} \times 5^{\prime}$. For comparison: the telescope's $F W H M$ is 14.4 . 
W. van Driel et al.: A neutral hydrogen survey of polar ring galaxies. IV.

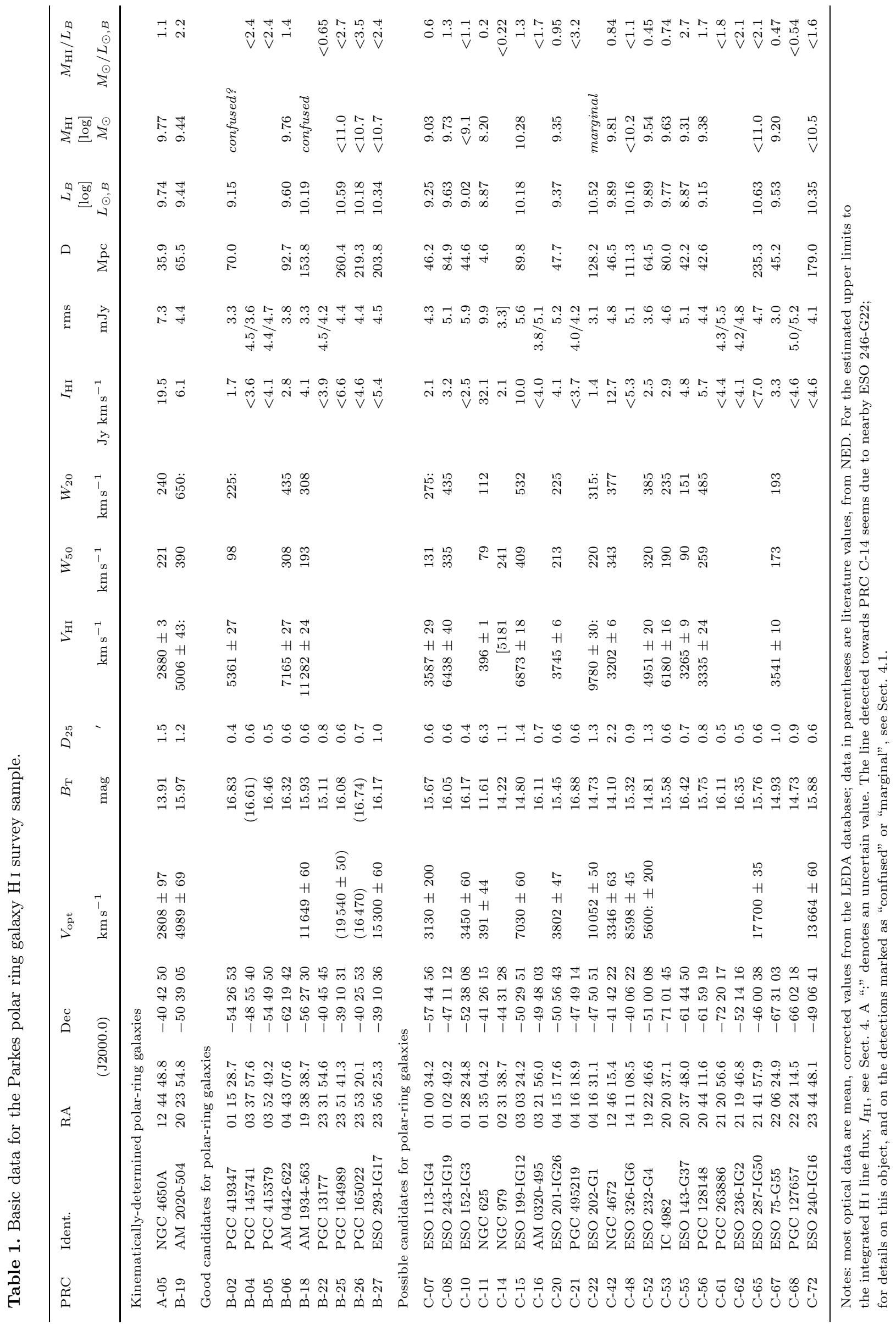


We calibrated, averaged and made a first baseline subtraction in our spectra using the standard multibeam receiver spectral line data reduction package available at the Parkes site, GRIDZILLA. The data were then reformatted, using a routine written by L.S. Staveley-Smith, for further reduction with the CLASS spectral line data reduction package. With CLASS we made a final, thirdorder polynomial baseline subtraction, and determined the global H I line parameters and rms. noise level in the spectra, after degrading the velocity resolution to $13.2 \mathrm{~km} \mathrm{~s}^{-1}$ for most spectra (see Sect. 4).

\section{Results}

The reduced Parkes 21-cm spectra are shown in Fig. 1 for all objects towards which line emission was detected, clearly or tentatively. For discussion of possible confusion with other galaxies in the beam, see Sect. 4.1 - we assumed that the emission line detected toward PRC C-14 is due to a nearby galaxy. Basic optical and $\mathrm{H}$ I line parameters of all observed objects are listed in Table 1. For the optical data we preferentially used the mean parameters from the LEDA database, or literature values from NED if these were not available (for the optical redshift of PRC B-18, see Sect. 4.1).

Columns in Table 1 are as follows: (1) galaxy number in the PRC, (2) identifications in other catalogues, (3 and 4) optical centre position, which was used as pointing position for the H I observations, (5) optical systemic velocity and its estimated uncertainty, if available, (6) the total blue magnitude, uncorrected for Galactic or internal extinction, (7) blue major axis diameter at the $25 \mathrm{mag} \operatorname{arcsec}^{-2}$ isophotal level, (8) centre velocity of the $\mathrm{H}$ I profile and its estimated uncertainty (see below), (9) width of the $\mathrm{H}$ I profile measured at the $50 \%$ level of the peak flux density, (10) idem, at the $20 \%$ level, (11) integrated H I line flux or an estimated $3 \sigma$ upper limit (see below), depending on the blue luminosity of the objects, if known (see text), (12) rms noise level of the spectrum; if two values are listed for an object, the first refers to the $\sim-1000$ to $12000 \mathrm{~km} \mathrm{~s}^{-1}$ velocity range and the second to the $\sim 8500$ to $21500 \mathrm{~km} \mathrm{~s}^{-1}$ range, (13) distance, derived preferably using heliocentric systemic $\mathrm{H}$ I line velocities, or otherwise optical velocities, corrected to the Local Standard of Rest following the prescription of Sandage \& Tammann (1981, RSA):

$$
\begin{aligned}
V_{\mathrm{LSR}}= & V_{\mathrm{hel}}-79 \cos l \cos b+296 \sin l \cos b \\
& -36 \sin b\left[\mathrm{~km} \mathrm{~s}^{-1}\right]
\end{aligned}
$$

and assuming a Hubble constant $H_{0}=75 \mathrm{~km} \mathrm{~s}^{-1} \mathrm{Mpc}^{-1}$, (14) blue luminosity, (15) H I mass, derived straight from the measured integrated $\mathrm{H}$ I line fluxes without any correction factors, such as for beam filling:

$M_{\mathrm{HI}}=2.356 \times 10^{5} D^{2} I_{\mathrm{HI}}\left[M_{\odot}\right]$

(16) ratio of the total H I mass and blue luminosity.

The instrumental velocity resolution of the reduced data is $13.2 \mathrm{~km} \mathrm{~s}^{-1}$, except for the narrow profile of C-11 $\left(6.6 \mathrm{~km} \mathrm{~s}^{-1}\right)$ and for the faint detection seen towards C-14 $\left(26.4 \mathrm{~km} \mathrm{~s}^{-1}\right)$. We estimated the uncertainties, $\sigma_{V_{\mathrm{HI}}}$, in the central H I velocities, $V_{\mathrm{HI}}$, following Fouqué et al. (1990):

$\sigma_{V_{\mathrm{HI}}}=4 R^{0.5} P_{\mathrm{W}}^{0.5} X^{-1}\left[\mathrm{~km} \mathrm{~s}^{-1}\right]$

where $R$ is the velocity resolution in $\mathrm{km} \mathrm{s}^{-1}, P_{W}=\left(W_{20^{-}}\right.$ $\left.W_{50}\right) / 2$ in $\mathrm{km} \mathrm{s}^{-1}$ and $X$ is the signal-to-noise ratio of a spectrum, which we defined as the ratio of the peak flux density and the rms noise. According to Fouqué et al., the uncertainty in the linewidths is $2 \sigma_{V_{\mathrm{HI}}}$ for $W_{50}$ and $3 \sigma_{V_{\mathrm{HI}}}$ for $W_{20}$.

As the H I linewidths of detected PRC objects show an increase with luminosity (van Driel et al., in prep.), though with large scatter, the estimated upper limits to the integrated $\mathrm{H}$ I line flux, $I_{\mathrm{HI}}$, are $3 \sigma$ values for flat-topped profiles with an estimated $W_{20}$ width depending on the blue luminosity of the galaxies, consistent with the upper limits listed in Paper III. For the 9 objects of unknown redshift, including C-14, a $W_{20}$ linewidth of $300 \mathrm{~km} \mathrm{~s}^{-1}$, was assumed, a typical value for the entire sample. For the objects not detected in the -1000 to $21500 \mathrm{~km} \mathrm{~s}^{-1}$ range, the average rms noise level over this entire range was used to estimate the upper limit. The resulting $M_{\mathrm{HI}}$ and $M_{\mathrm{HI}} / L_{B}$ upper limits for the objects without known redshift are obviously only significant if their redshift in fact lies within the velocity range searched.

\subsection{Notes on individual objects}

The data on possible companions that may confuse the Parkes spectra were compiled using the online NED and LEDA databases, searching in an area of $22^{\prime}$ diameter (i.e., 1.5 times the telescope's $F W H M$ ) centered on the pointing positions of the telescope (see Table 1). The published H I data on our target PRC objects are summarized and compared to our present Parkes results in Table 2.

\section{Kinematically-determined polar-ring galaxies}

A-05 (=NGC 4650A): this is probably the beststudied of all polar ring galaxies, see, e.g., Arnaboldi et al. (1997). Detected in the present survey and in 4 others, including two interferometric mappings (see Table 2). The VLA and Australia Telescope H I imaging show that our single-dish spectrum is not confused by the two other relatively bright, but early-type galaxies in the Parkes beam, NGC $4650\left(V_{\mathrm{opt}}=2859 \mathrm{~km} \mathrm{~s}^{-1}\right)$ and NGC 4650B $\left(V_{\mathrm{opt}}=2515 \mathrm{~km} \mathrm{~s}^{-1}\right)$. These early-type galaxies are expected to be gas-poor

B-19 (= AM 2020-504): this is the best-studied case of a polar ring around an elliptical galaxy (Arnaboldi et al. 1993a); the other case in our sample is PRC C-42, see below. The polar ring shows a gentle warp and is probably stable, giving time to stars to form and grow old. It has a UV spectrum typical of a starburst galaxy (Arnaboldi et al. 1993b). The object was detected in the present survey, at $V_{\mathrm{HI}}=5006 \pm 43 \mathrm{~km} \mathrm{~s}^{-1}$. Our profile does not seem to be confused by another object in the beam. The H I line velocity corresponds well to the mean optical value of the PRC object, $4989 \pm 69 \mathrm{~km} \mathrm{~s}^{-1}$ (LEDA). 

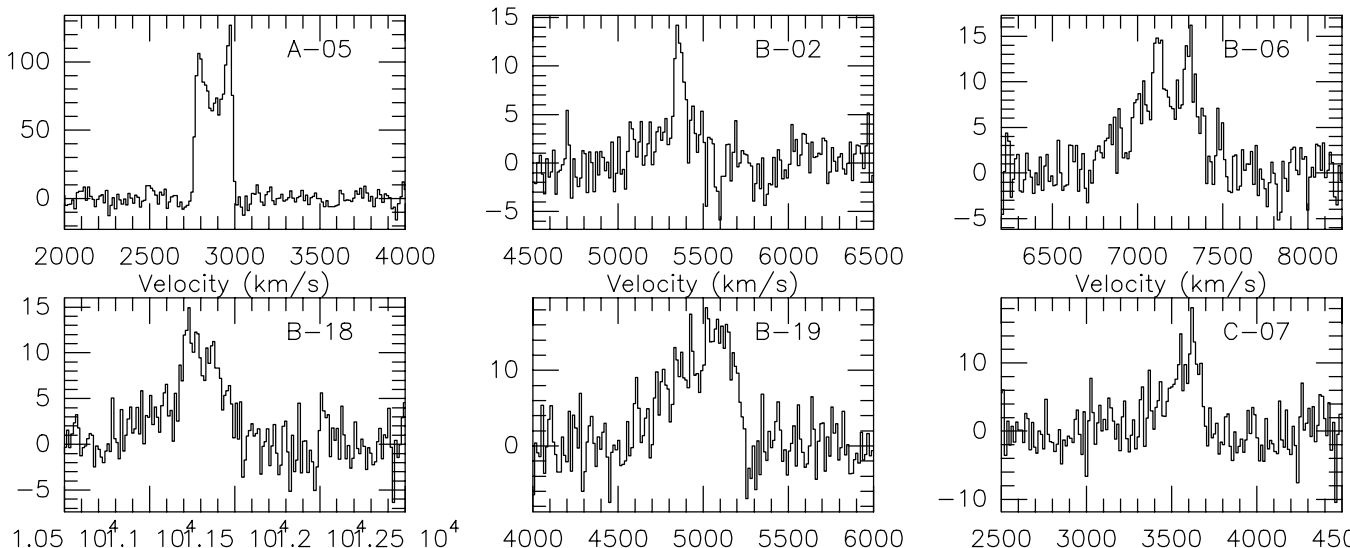

6500700075008000
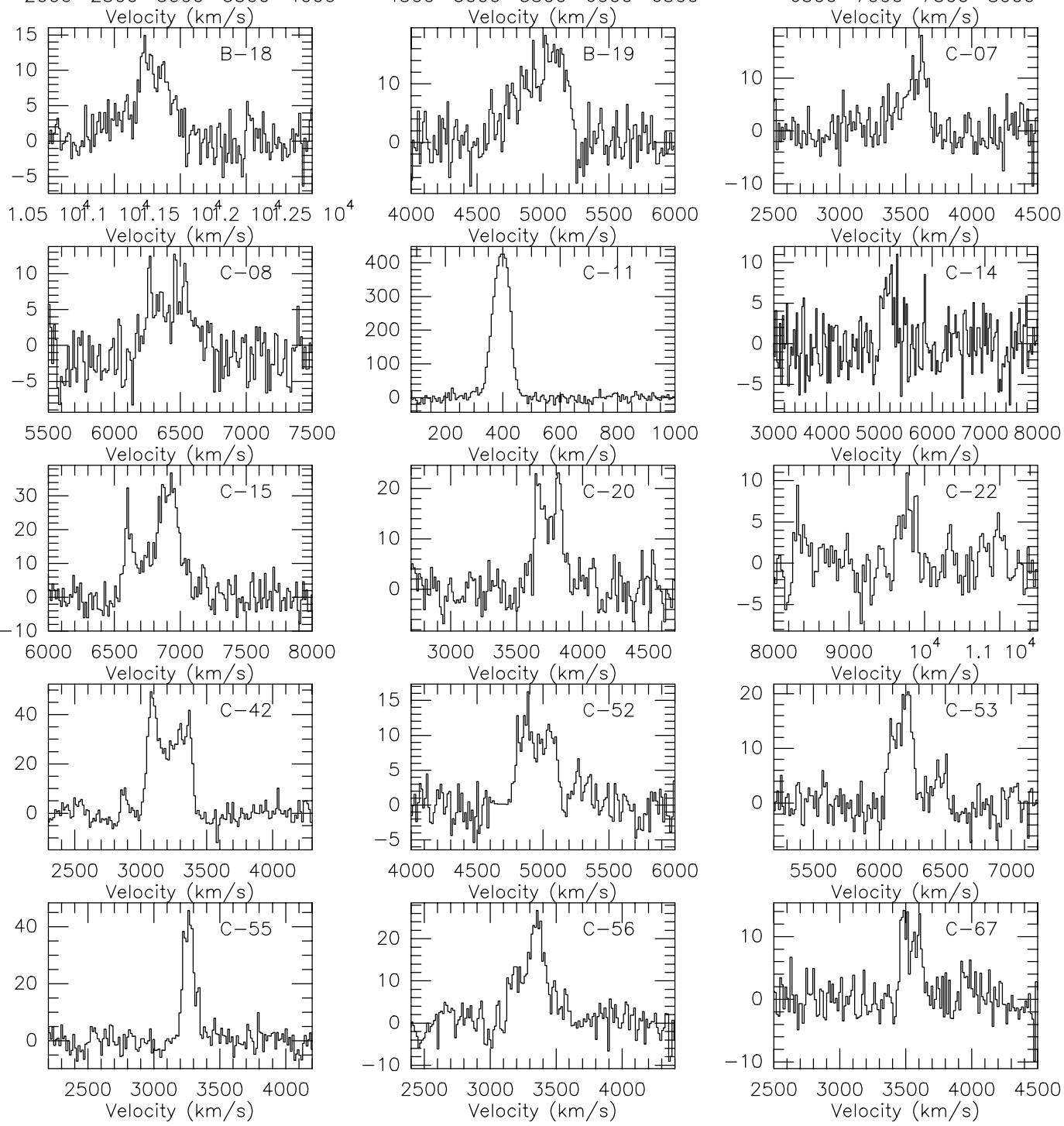

Fig. 2. Parkes 21-cm H I line spectra of all clear and marginal detections. Each galaxy is identified by its PRC number. The axes are heliocentric velocity in $\mathrm{km} \mathrm{s}^{-1}$, and flux density in $\mathrm{mJy}$. The velocity resolution of the spectra is $13.2 \mathrm{~km} \mathrm{~s}{ }^{-1}$, except for $\mathrm{C}-11\left(6.6 \mathrm{~km} \mathrm{~s}^{-1}\right)$ and $\mathrm{C}-14\left(26.4 \mathrm{~km} \mathrm{~s}^{-1}\right)$.

Just outside the telescope's $F W H M$ radius, two galaxies lie at about $8^{\prime}$ distance from the PRC target: the quite face-on $(b / a=0.8) 14.0 \mathrm{mag}$ Sc type spiral ESO 234-G016 at $V_{\mathrm{opt}}=5215 \pm 82 \mathrm{~km} \mathrm{~s}^{-1}$ and the $14.6 \mathrm{mag} \mathrm{Sbc}$ type spiral ESO 234-G017, of unknown redshift. Given the difference in optical velocities $\left(225 \mathrm{~km} \mathrm{~s}^{-1}\right)$ and the range of velocities in our H I profile, it seems unlikely that the Parkes profile was confused by ESO 234-G016.

\section{Good candidates}

B-02 (= PGC 419347): this object does not have a known optical redshift. We detected an H I profile towards it, at $V_{\mathrm{HI}}=5361 \pm 27 \mathrm{~km} \mathrm{~s}^{-1}$. At 7.3 distance, around the telescope's $F W H M$ radius, lies 15.5 mag Sc? type spiral ESO 151-G37, with $V_{\text {opt }}=5452 \pm 60 \mathrm{~km} \mathrm{~s}^{-1}$ (Mathewson \& Ford 1996), $91 \mathrm{~km} \mathrm{~s}^{-1}$ higher than the $\mathrm{HI}$ value. If the detection were in fact entirely due to ESO 151-G37, its integrated $\mathrm{HI}$ line flux would be $\sim 3.4 \mathrm{Jy} \mathrm{km} \mathrm{s}^{-1}$, at $\sim 50 \%$ beam efficiency, implying an $M_{\mathrm{HI}} / L_{B}$ ratio of $0.8 M_{\odot} / L_{\odot, B}$. Such a ratio is about 2.5 times the average for Sc spirals (Roberts \& Haynes 1994) and therefore highly unlikely. If, on the other hand, all $\mathrm{H}$ I were to be due to B-02 alone, its $M_{\mathrm{HI}} / L_{B}$ ratio would be $1.4 M_{\odot} / L_{\odot, B}$, 
Table 2. Published H I data for the Parkes PRG sample.

\begin{tabular}{llrrrrl}
\hline PRC & $\begin{array}{l}\text { Tel. } \\
\text { code }\end{array}$ & $\begin{array}{r}V_{\mathrm{HI}} \\
\mathrm{km} \mathrm{s}^{-1}\end{array}$ & $\begin{array}{r}I_{\mathrm{HI}} \\
\mathrm{Jy} \mathrm{km} \mathrm{s}^{-1}\end{array}$ & $\begin{array}{r}W_{50} \\
\mathrm{~km} \mathrm{~s}^{-1}\end{array}$ & $\begin{array}{r}W_{20} \\
\mathrm{~km} \mathrm{~s}^{-1}\end{array}$ & Ref. \\
\hline A-05 & $\mathrm{P}$ & 2880 & 19.5 & 221 & 240 & $*$ \\
& $\mathrm{P}$ & 2910 & & & 220 & Ba97 \\
& G43 & 2909 & 23.2 & 244 & & $\mathrm{Ri94}$ \\
& $\mathrm{V}$ & 2910 & 16.0 & 231 & 243 & $\mathrm{vG87}$ \\
& AT & 2905 & 23.1 & 221 & 240 & Ar97 \\
C-11 & $\mathrm{P}$ & 396 & 32.1 & 79 & 112 & $*$ \\
& $\mathrm{P}$ & 387 & 38.0 & & 116 & Re82 \\
& $\mathrm{P}$ & 387 & 26.0 & & & Bo88 \\
& G43 & 404 & 32.4 & & & FT81 \\
& G43 & 394 & 37.4 & 113 & & Ri94 \\
& IAR & 370 & 35.1 & 80 & 103 & BM85 \\
& V & 406 & 30.6 & & & Co00 \\
C-14 & P & {$[5141$} & 2.1 & 241 & $241]$ & $*$ \\
& P & & $<10.4$ & & & Ha81 \\
& G43 & 4775 & 26.2 & $678:$ & & Ri94 \\
C-42 & P & 3202 & 12.7 & 343 & 377 & $*$ \\
& P & 3289 & 15.6 & 356 & 403 & Aa89 \\
& G43 & 3242 & 7.7 & 401 & & Ri94 \\
\hline
\end{tabular}

Telescope codes:

$\begin{array}{ll}\text { AT } & \text { Australia Telescope } \\ \text { G43 } & \text { Green Bank 43-m } \\ \text { IAR } & \text { I.A.R. 30-m } \\ \text { P } & \text { Parkes 64-m } \\ \text { V } & \text { VLA }\end{array}$

References:

Aa89 Aaronson et al. (1989)

Ar97 Arnaboldi et al. (1997)

BM85 Bajaja \& Martin (1985)

Ba97 Barnes et al. (1997)

Bo88 Boissé et al. (1988)

Co00 Côté et al. (2000)

FT81 Fisher \& Tully (1981)

Ha81 Hawarden et al. (1981)

Re82 Reif et al. (1982)

Ri94 Richter et al. (1994)

vG87 van Gorkom et al. (1987)

$\star \quad$ this paper

a quite common value for an object from PRC Category B. A long-slit H $\alpha$ spectrum of ESO 151-G37 (Mathewson \& Ford 1996) shows a maximum rotational velocity, uncorrected for inclination, of $107 \mathrm{~km} \mathrm{~s}^{-1}$, much larger than half the $W_{50}$ width of our H I spectrum, $45 \mathrm{~km} \mathrm{~s}^{-1}$. In conclusion, it is not very likely that our $\mathrm{H}$ I detection may be confused by nearby ESO 151-G37.

B-06 (= AM 0442-622): detected in the present survey, at $V_{\mathrm{HI}}=7165 \mathrm{~km} \mathrm{~s}^{-1}$. The only other catalogued galaxy within the telescope's FWHM, the low surface brightness object LSBG F118-043, of unknown redshift and $B_{\mathrm{T}}$ 18.7 mag. Though low surface brightness galaxies can be quite gas-rich, it is $\sim 2.4$ mag fainter than B-06 in the blue, and hence not a likely candidate for confusion with our H I profile. The $M_{\mathrm{HI}} / L_{B}$ ratio of $1.4 M_{\odot} / L_{\odot, B}$ derived for B-06 assuming that all detected gas resides in it is a quite common value among the PRC Category B objects.

B-18 (= AM 1934-563): detected in the present survey, at $V_{\mathrm{HI}}=11282 \pm 24 \mathrm{~km} \mathrm{~s}^{-1}$, with a $W_{50}$ width of $193 \mathrm{~km} \mathrm{~s}^{-1}$. Its nuclear $\mathrm{H} \alpha$ and [NII] emission lines (Reshetnikov et al. 2001) indicate fast rotation of the gas in the innermost regions, reaching a projected rotational velocity of $\sim 230 \mathrm{~km} \mathrm{~s}^{-1}$ at $\sim 2^{\prime \prime}$ from the nucleus, as well as signs of Sy2 or LINER activity. Its mean optical systemic velocity listed in LEDA, $11703 \pm 153 \mathrm{~km} \mathrm{~s}^{-1}$, did not take into account the latter two of the four following published redshifts: $11556 \pm 48 \mathrm{~km} \mathrm{~s}^{-1}$ (Fisher et al. 1995), $11842 \pm$ $120 \mathrm{~km} \mathrm{~s}^{-1}$ (Allen et al. 1991), $11850 \pm 200 \mathrm{~km} \mathrm{~s}^{-1}$ (Fairall \& Jones 1991) and $11613 \pm 54 \mathrm{~km} \mathrm{~s}^{-1}$ (Reshetnikov et al. 2001). From these three values, we derive a weighted mean value of $V_{\mathrm{opt}}=11649 \pm 60 \mathrm{~km} \mathrm{~s}^{-1}, 367 \mathrm{~km} \mathrm{~s}^{-1}\left(\sim 6 \sigma_{V_{\mathrm{opt}}}\right)$ lower than the $\mathrm{H}$ I value, a significantly large difference. Three galaxies of similar size and magnitude can be seen in Fig. 1, which appear to form a triplet. Besides the PRC object, these are PGC $399718\left(B_{\mathrm{T}}=15.7\right.$ and $\left.D_{25}=0.7\right)$ and PGC $400092\left(B_{\mathrm{T}}=16.2\right.$ and $\left.D_{25}=0.5\right)$, both of unknown redshift. The $M_{\mathrm{HI}} / L_{B}$ ratio of $1.5 M_{\odot} / L_{\odot, B}$ we would derive for B-18 if all $\mathrm{HI}$ were concentrated in it is quite common for a PRC Category B object, however. In conclusion, we cannot exclude that the $\mathrm{H}$ i profile may be confused with, or due to, another member of the triplet.

B-27 (= ESO 293-IG17): not detected in the present survey. Our Green Bank H I survey (Paper I) did not cover the optical redshift of the object $\left(15300 \mathrm{~km} \mathrm{~s}^{-1}\right)$, which was published afterwards.

\section{Possible PRG candidates}

C-07 (= ESO 113-IG4): an H I line was detected in the present survey, at $V_{\mathrm{HI}}=3587 \pm 29 \mathrm{~km} \mathrm{~s}^{-1}$. The optical systemic velocity, $3130 \mathrm{kms}^{-1}$ (from Keel 1985), is $477 \mathrm{~km} \mathrm{~s}^{-1}$ lower than the Hi value. Though its uncertainty is listed as $35 \mathrm{kms}^{-1}$ in LEDA, a value of $200 \mathrm{~km} \mathrm{~s}^{-1}$ was quoted in Keel's paper - we adopted the latter uncertainty. Given the relatively large uncertainties involved, the $\mathrm{H}$ I and optical velocities may be consistent. No candidates for confusion with our H I spectrum were found within the search area.

C-08 (= ESO 243-IG19): detected in the present survey, at $V_{\mathrm{HI}}=6438 \pm 40 \mathrm{~km} \mathrm{~s}^{-1}$. No optical redshift is known of this object. No candidates for confusion with our H I spectrum were found within the search area.

C-11 (= NGC 625): detected in the present survey and in 6 others (see Table 2), with good agreement between the global H i line parameters measured at different telescopes and in different studies. The galaxy was mapped in $\mathrm{H}$ I at the VLA (Côté et al. 2000), where a peculiar gas distribution and kinematics was found. The $\mathrm{HI}$ is rotating around the optical major axis, rather than the minor axis, though its distribution is elongated along the major axis - contrary to the situation in polar rings. Multiple velocity peaks occur at many places in the VLA data. Consequently no $\mathrm{H}$ I rotation curve could be derived. The ionized gas in the galaxy also follows complex orbits 
(Marlowe et al. 1997), which do not match the Hi kinematics, however. It is a rather red system $(B-R)=0.89$, with a smooth morphology reminiscent of lenticular galaxies, and strong emission lines, like a blue compact dwarf (Skillman et al. 2002) According to Côté et al., the seriously disturbed nature of NGC 625 is due to a major merger event rather than a case of bad digestion of an accreted $\mathrm{H}$ I cloud.

C-14 (= NGC 979): No optical velocity is known of this object, and it does not appear to have been detected in Hi. A detection with the Green Bank 43-m telescope was reported in Paper I, with a centre velocity of $4775 \mathrm{~km} \mathrm{~s}^{-1}$, an exceptionally large width of $W_{20}=678 \mathrm{~km} \mathrm{~s}^{-1}$ and a peak flux density of $\sim 30 \mathrm{mJy}$, while at Parkes no line signal was detected by Hawarden et al. (1981), with a published upper limit to the integrated $\mathrm{H} \mathrm{I}$ line flux of $10.4 \mathrm{Jy} \mathrm{km} \mathrm{s}^{-1}$. As no trace of the signal seen at Green Bank can be found in our Parkes data, which have an rms noise level of $3.3 \mathrm{mJy}$, it must have been due to interference. Our Parkes spectrum shows a marginal $\mathrm{H}$ I detection at $V_{\mathrm{HI}}=5240 \mathrm{~km} \mathrm{~s}^{-1}$, which is likely to be associated with ESO 246 G-22, a 14.4 mag SBc spiral with an optical redshift of $5127 \pm 60 \mathrm{~km} \mathrm{~s}^{-1}$ (LEDA), 9'1 from the PRC object. We therefore consider PRC C-14 to be undetected in our survey, and the upper limit to its $M_{\mathrm{HI}} / L_{B}$ ratio of $0.22 M_{\odot} / L_{\odot, B}$ listed in Table 1 was estimated using a $300 \mathrm{~km} \mathrm{~s}^{-1}$ linewidth, like for all objects of unknown redshift.

C-15 (= ESO199-12): A strong H I line was detected in the present survey, with $V_{\mathrm{HI}}=6873 \pm 18 \mathrm{~km} \mathrm{~s}^{-1}$ and $W_{50}=409 \mathrm{~km} \mathrm{~s}^{-1}$. There appears to be a faint detection in the Parkes HIPASS survey data, at $\sim 7000 \mathrm{~km} \mathrm{~s}^{-1}$, with a peak flux density of about $30 \mathrm{mJy}$, like in our spectrum. The large uncertainty in the optical velocity of the object, as listed in LEDA, $6727 \pm 358 \mathrm{~km} \mathrm{~s}^{-1}$, is due to one discrepant measurement: $6296 \pm 300 \mathrm{~km} \mathrm{~s}^{-1}$ (Kirhakos \& Steiner 1990), compared to the $7033 \pm 35$ and $7026 \pm 75: \mathrm{km} \mathrm{s}^{-1}$ from, respectively, Da Costa et al. (1991) and Chincarini et al. (1984). Therefore, the optical redshift is rather $7030 \pm 60 \mathrm{~km} \mathrm{~s}^{-1}, 157 \mathrm{~km} \mathrm{~s}^{-1}$ (i.e., $2.5 \sigma_{V_{\mathrm{opt}}}$ ) higher than the $\mathrm{H}$ I value. The only other galaxy of significant size $\left(D_{25}=1.2\right)$ within the telescope's $F W H M$ is IC 1877, an edge-on Sb:pec spiral of unknown redshift and $B_{\mathrm{T}} 16.2 \mathrm{mag}$. It does not seem likely that this 1.4 mag fainter object would cause serious confusion in the H I spectrum of the target object. The $M_{\mathrm{HI}} / L_{B}$ ratio of $1.25 M_{\odot} / L_{\odot, B}$ we would derive if all $\mathrm{H}$ I was concentrated in the PRC object is not uncommon for PRC category-C objects.

C-16 (= AM 0320-495): our Parkes spectrum only shows an off-beam detection of an unidentified galaxy, with $V_{\mathrm{HI}}=1023 \mathrm{~km} \mathrm{~s}^{-1}, W_{50}=207 \mathrm{~km} \mathrm{~s}^{-1}$ and $I_{\mathrm{HI}}=$ $-2.1 \mathrm{Jy} \mathrm{km} \mathrm{s}^{-1}$. It appears to be a sidelobe-detection of $B_{\mathrm{T}} 13.6 \mathrm{mag}$, Scd spiral IC 1914, at 27.3 distance from the target object. Observations of IC 1914 at Parkes by Longmore et al. (1982) show $V_{\mathrm{HI}}=1037 \mathrm{~km} \mathrm{~s}^{-1}$, $W_{50}=206 \mathrm{~km} \mathrm{~s}^{-1}$ and $I_{\mathrm{HI}}=53.0 \mathrm{Jy} \mathrm{km} \mathrm{s}{ }^{-1}$.
C-20 (= ESO 201-IG26): detected in the present survey, at $V_{\mathrm{HI}}=3745 \pm 6 \mathrm{~km} \mathrm{~s}^{-1}$, corresponding well with the optical systemic velocity of $3802 \pm 47$ (LEDA). Also in, the less sensitive, Parkes HIPASS data a tentative detection is seen at the same velocity. No candidates for confusion with our $\mathrm{H}$ I spectrum were found within the search area: at 2'.4 distance, low surface brightness object LSBG F202-064, of unknown redshift and $B_{\mathrm{T}} 17.7 \mathrm{mag}$, does not seem a likely candidate: though low surface brightness galaxies can be quite gas-rich, it is $\sim 2.2$ mag fainter than C-20. The $M_{\mathrm{HI}} / L_{B}$ ratio of $0.95 M_{\odot} / L_{\odot, B}$ derived for $\mathrm{C}-20$ assuming that all detected gas resides in it is a quite common value for a PRC Category $\mathrm{C}$ objects.

C-22 (= ESO 202-G1): in our present survey no H I line is seen at the optical velocity, $10052 \pm 50 \mathrm{~km} \mathrm{~s}^{-1}$ (LEDA; based on 2 published values), while a tentative line signal is seen at $\sim 9780 \pm 30 \mathrm{~km} \mathrm{~s}^{-1}$, with $W_{50}=$ $220 \mathrm{~km} \mathrm{~s}^{-1}$ and $I_{\mathrm{HI}}=1.4 \mathrm{Jy} \mathrm{km} \mathrm{s}{ }^{-1}$. Though no candidates for confusion with our $\mathrm{HI}$ spectrum were found within the search area, we cannot be certain that the object was detected, seen the weakness of the tentative line detections and the difference of $272 \mathrm{~km} \mathrm{~s}^{-1}$ (i.e., $\left.5.5 \sigma_{V_{\text {opt }}}\right)$ between the well-established optical redshift and our $\mathrm{H} \mathrm{I}$ value. We have therefore marked the spectral line as "marginal" in Table 2, and we did not derive an $\mathrm{HI}$ mass from it.

C-42 (= NGC 4672): it has an elliptical stellar core rotating perpendicularly to the disk and appears to be the end result of the accretion of material in polar orbits in a disk around a pre-existing oblate spheroid, like in the prototype PRC B-19 (Sarzi et al. 2000). The object was detected in the present survey, at $V_{\mathrm{HI}}=3202 \pm 6 \mathrm{~km} \mathrm{~s}^{-1}$, as well as previously at Parkes and at Green Bank (see Table 2) at similar velocities, which are all consistent with the mean optical systemic value of $3346 \pm 63 \mathrm{~km} \mathrm{~s}^{-1}$ (LEDA). The integrated line flux of $7.7 \mathrm{Jy} \mathrm{km} \mathrm{s}^{-1}$ measured at Green Bank (Paper I) is significantly lower than the 12.7-15.6 $\mathrm{Jy} \mathrm{km} \mathrm{s}^{-1}$ found at Parkes (Aaronson et al. 1989 , and the present paper). No candidates for confusion with our H I spectrum were found within the search area: though NGC 4677, a 13.9 mag object at 10'7 distance, has a velocity of $3135 \pm 40 \mathrm{~km} \mathrm{~s}^{-1}$ (LEDA), similar to that of the PRC object, it is well outside the telescope's $F W H M$ and classified as SB0 and therefore expected to be gas-poor.

C-48 (= ESO 326-IG6): not detected in the present survey, nor in our Green Bank survey (Paper I), with an almost two times higher rms noise level of $9.5 \mathrm{mJy}$.

C-52 (= ESO 232-G4): detected in the present survey, at $V_{\mathrm{HI}}=4951 \pm 20 \mathrm{~km} \mathrm{~s}^{-1}$. Its published optical systemic velocities are $5083 \pm 57 \mathrm{~km} \mathrm{~s}^{-1}$ (Reshetnikov et al. 2001), based on faint lines, and 5600: $\pm 200 \mathrm{~km} \mathrm{~s}^{-1}$ (Fairall 1984), a rather uncertain value. Seen the uncertainties, these values may be consistent with the H I value. Its nuclear spectrum was tentatively classified as LINER: by Reshetnikov et al. (2001). No candidates for confusion with our H I spectrum were found within the search area. 
C-53 (= IC 4982): detected in the present survey, at $V_{\mathrm{HI}}=6180 \pm 16 \mathrm{~km} \mathrm{~s}^{-1}$. No object is visible on the DSS at the position given in the PRC, $20^{\mathrm{h}} 15^{\mathrm{m}} 22^{\mathrm{s}} .4,-71^{\circ} 11^{\prime} 13^{\prime \prime}$ (B1950.0); the galaxy identified as C-53 in Fig. 2 in the PRC is actually IC 4982, 1'.9 NW of the C-53 position. We therefore used the optical position of IC 4982 as our pointing centre at Parkes and listed its optical characteristics in Table 1. No candidates for confusion with our H I spectrum were found within the search area: IC 4985, 2 '. NE of the target object is a $14.8 \mathrm{mag}$ S0 galaxy at a quite different redshift of $V_{\text {opt }}=4442 \mathrm{~km} \mathrm{~s}^{-1}$ (LEDA), and expected to be gas-poor.

C-55 (= ESO 143-G37): detected in the present survey, at $V_{\mathrm{HI}}=3265 \pm 9 \mathrm{~km} \mathrm{~s}^{-1}$. The HIPASS data show an H I detection similar to ours. No candidates for confusion with our H I spectrum were found within the search area: no data are available for the small galaxy pair AM2033620 at 6.2 from the target object.

C-56 (= PGC 128148): detected in the present survey, at $V_{\mathrm{HI}}=6873 \pm 18 \mathrm{~km} \mathrm{~s}^{-1}$. No candidates for confusion with our H I spectrum were found within the search area.

C-67 (= ESO 75-G55): detected in the present survey, at $V_{\mathrm{HI}}=6873 \pm 18 \mathrm{~km} \mathrm{~s}^{-1}$. No candidates for confusion with our H I spectrum were found within the search area.

\section{Conclusions}

Of the 18 PRC objects towards which $\mathrm{H}$ I line emission was noted, confusion with one or more galaxies in the telescope beam is suspected, though with varying degrees of probability, in three cases (PRC B-02, B-18 and C-14), while one weak $\mathrm{HI}$ line (of PRC C-22) is regarded as a tentative detection only, seen the significant difference between the $\mathrm{H}$ I and optical velocities. A previously reported detection of PRC C-14, apparently caused by radio interference, was not reconfirmed. Comparing with literature values as well as HIPASS data, the following eight objects were detected for the first time in $\mathrm{H}$ I: PRC B-06, B-19, C-07, C-08, C-53, C-55, C-56 and C-67. Of the 33 observed galaxies 22 now have known radial velocities, five of which were determined here for the following objects with previously unknown redshifts: PRC B-06, C-08, C-53, C-56 and C-67.

Acknowledgements. We are grateful to the ATNF staff at the Parkes observatory and in Epping for their assistance with the observations and data reduction. The Parkes Telescope is part of the Australia Telescope, which is funded by the Commonwealth of Australia for operation as a national facility managed by CSIRO. This research has made use of the NASA/IPAC Extragalactic Database (NED), which is operated by the Jet Propulsion Laboratory, California Institute of Technology, under contract with the National Aeronautics and Space Administration and the Lyon-Meudon Extragalactic Database (LEDA). WvD acknowledges the financial support of the ASTE of INSU for the observations at Parkes.

\section{References}

Aaronson, M., Bothun, G. D., Cornell, M. E., et al. 1989, ApJ, 338,654

Allen, D. A., Norris, R. P., Meadows, V. S., \& Roche, P. F. 1991, MNRAS, 248, 528

Arnaboldi, M., Capaccioli, M., Cappellaro, E., et al. 1993a, A\&A, 26721

Arnaboldi, M., Barbaro, G., Buson, L., et al. 1993b, A\&A, 268, 103

Arnaboldi, M., Oosterloo, T. A., Combes, F., Freeman, K. C., \& Koribalski, B. 1997, AJ, 113, 585

Bajaja, E., \& Martin, M. C. 1985, AJ, 90, 1783

Barnes, L., Staveley-Smith, L., Webster, R. L., \& Walsh, W. 1997, MNRAS, 288, 307

Boissé, P., Dickey, J. M., Kazès, I., \& Bergeron, J. 1988, A\&A, 191, 193

Brocca, C., Bettoni, D., \& Galletta, G. 1997, A\&A, 326, 907

Chincarini, G.,Tarenghi, M., Sol, H., et al. 1984, A\&AS, 57, 1

Côté, S., Carignan, C., \& Freeman, K. C. 2000, AJ, 120, 3027

Da Costa, L. N., Pellegrini, P. S., Davis, M., et al. 1991, ApJS, 75,935

Fairall, A. P. 1984, MNRAS, 210, 69

Fairall, A., \& Jones, A. 1991, Southern Redshifts Catalogue (Pub. of the Dept. of Astronomy, Univ. of Cape Town), 11

Fisher, J. R., \& Tully, R. B. 1981, ApJS, 47, 139

Fisher, J. R., Huchra, J. P., Strauss, M. A., et al. 1995, ApJS, 100, 69

Fouqué, P., Bottinelli, L., Durand, N., Gouguenheim L., \& Paturel, G. 1990, A\&AS, 86, 473

Hawarden, T. G., Longmore, A. J., Goss, W. M., Mebold, U., \& Tritton, S. B. 1981, MNRAS, 196, 175

Huchtmeier, W. 1997, A\&A, 319, 401 (Paper II)

Keel, W. C. 1985, AJ, 90, 2207

Kirhakos, S. D., \& Steiner, J. E. 1990, AJ, 99, 1722

Krumm, N., \& Salpeter, E. E. 1976, ApJ, 208, L7

Longmore, A. J., Hawarden, T. G., Goss, W. M., Mebold, U., \& Tritton, S. B. 1982, MNRAS, 200, 325

Marlowe, A., Meurer, G., Heckman, T., \& Schommer, R. 1997, ApJS, 112, 285

Mathewson, D. S., \& Ford, V. L. 1996, ApJS, 107, 97

Reif, K., Mebold, U., Goss, W. M., van Woerden, H., \& Siegman, B. 1982, A\&AS, 50, 451

Reshetnikov, V. P., \& Sotnikova, N. 1997, A\&A, 325, 933

Reshetnikov, V. P., Faúndez-Abans, M., \& de Oliveira-Abans, M. 2001, MNRAS, 322, 689

Richter, O.-G., Sackett, P. D., \& Sparke, L. S. 1994, AJ, 107, 99 (Paper I)

Roberts, M. S., \& Haynes, M. P. 1994, ARA\&A, 32, 115

Sandage, A., \& Tammann, R. A. 1981, A Revised Shapley Ames-Catalog of Bright Galaxies, Carnegie Inst. of Washington (RSA)

Sarzi, M., Corsini, E. M., Pizzella, A., et al. 2000, A\&A, 360, 439

Skillman, E., Côté, S., \& Miller, B. 2002, AJ, in press

Staveley-Smith, L., Wilson, W. E., Bird, T. S., et al. 1996, PASA, 13, 243

van Driel, W., Arnaboldi, M., Combes, F., \& Sparke, L. S. 2000, A\&A, 141, 385 (Paper III)

van Gorkom, J. H., Schechter, P., \& Kristian, J. 1987, ApJ, 314,457

Whitmore, B. C., Lucas, R. A., McElroy, D. B., et al. 1990, AJ, 100, 1489 (PRC)

Whitmore, B. C. 1991, in Warped Disks and Inclined Rings around Galaxies, ed. S. Casertano, P. Sackett, \& F. Briggs (Cambridge University press: Cambridge), 60 